Meta

Journal des traducteurs

Translators' Journal

\title{
Clefs pour une histoire de la traductologie soviétique
}

\section{Christian Balliu}

Volume 50, numéro 3, août 2005

Le prisme de l'histoire

The History Lens

URI : https://id.erudit.org/iderudit/011605ar

DOI : https://doi.org/10.7202/011605ar

Aller au sommaire du numéro

Éditeur(s)

Les Presses de l'Université de Montréal

ISSN

0026-0452 (imprimé)

1492-1421 (numérique)

Découvrir la revue

Citer cet article

Balliu, C. (2005). Clefs pour une histoire de la traductologie soviétique. Meta, 50(3), 934-948. https://doi.org/10.7202/011605ar

\section{Résumé de l'article}

La traduction est une activité sociologiquement déterminée. On peut dire qu'une traductologie « nationale " s'est développée en Union soviétique, avec une véritable planification des oeuvres à traduire. Cet article s'attachera à poser les jalons historiques de cette recherche théorique. d'utilisation que vous pouvez consulter en ligne.

https://apropos.erudit.org/fr/usagers/politique-dutilisation/ 


\title{
Clefs pour une histoire de la traductologie soviétique
}

\author{
CHRISTIAN BALLIU \\ ISTI - Haute École de Bruxelles, Bruxelles, Belgique \\ ISIT, Paris, France \\ cballiu@heb.be
}

\section{RÉSUMÉ}

La traduction est une activité sociologiquement déterminée. On peut dire qu'une traductologie «nationale » s'est développée en Union soviétique, avec une véritable planification des œuvres à traduire. Cet article s'attachera à poser les jalons historiques de cette recherche théorique.

\section{ABSTRACT}

Translation is a sociologically-related activity. Translation studies can be said to have developed on a national level in the Soviet Union, with central planning of works to be translated. In the present article, an attempt will be made to put this theoretical research in a historical perspective.

\section{MOTS-CLÉS/KEYWORDS}

traductologie, Union soviétique, planification, équivalence, linguistique

«Seul le nom distingue le traducteur de l'auteur»

V. Trediakovsky

La traductologie russe du $\mathrm{xx}^{\mathrm{e}}$ siècle s'est indubitablement développée dans le contexte de l'Union soviétique. Ses axes de réflexion et de recherche gravitent autour de la notion de «traduisibilité $»^{1}$ (переводимость). Dès les années 1920, les théoriciens russes de la traduction accordent une place prépondérante à l'étude de cette notion, car la traduction était à l'époque souvent considérée comme une tâche irréalisable, un objectif inaccessible en l'absence d'une exactitude et d'une fidélité absolues: ce que nous appellerions en termes plus contemporains l'«entropie».

En effet, la fidélité se définissait comme la transmission de l'intégralité des éléments formels de l'original et la préservation de ses fonctions sémantiques et stylistiques. Cette antinomie - cette aporie, pourrait-on dire - entrava l'essor de la traduction dans la jeune Union soviétique ${ }^{2}$. On pourrait citer à titre d'exemple le cas de Valéri Brioussov, remarquable poète symboliste russe. Connu pour ses traductions de la poésie française contemporaine et celles d'Émile Verhaeren, il traduisit aussi Virgile, Goethe et E. A. Poe. Le littéralisme de Brioussov ${ }^{3}$, caractéristique de sa deuxième période, rompt complètement avec les plus belles traductions russes du $\mathrm{XIX}^{\mathrm{e}}$ siècle $^{4}$ et va incontestablement freiner la traduction libre jusque dans les années 1930.

Toutefois, dans les années 1930, les théoriciens accordèrent une attention plus grande à une notion nouvelle, la «correspondance fonctionnelle» (Функциональное соответствие), laquelle nécessite des écarts formels par rapport à l’original; mais la 
pratique ne suivit pas toujours la voie tracée. Pour sortir la traduction de cette impasse théorique, il fallut insister sur le statut polymorphe de l'original, sur l'unité indissoluble de la forme et du contenu.

La première leçon, non négligeable, fut tirée par Maxime Gorki. Le fondateur du réalisme socialiste ${ }^{5}$ était également rédacteur, critique et directeur de traductions. En bon héritier de la littérature russe classique, il décida d'élargir l'éventail des compétences demandées au traducteur et insista sur la nécessité de conjuguer connaissances linguistiques et formation générale solide. Ce fonds commun se retrouve déjà chez ces grands intellectuels et critiques littéraires que sont Belinsky, Tchernychevsky et autres Dobrolioubov. Cela est tout à fait normal, puisque la période 1860-1914 est le théâtre en Russie d'une critique «large», englobant des réflexions journalistiques, des essais théoriques ou encore des études universitaires. En d'autres termes, la traduction deviendra à partir des années 1930 une discipline justiciable d'un enseignement spécialisé de niveau universitaire, bien avant la prise de conscience occidentale des décennies 1950-19606.

Il faut dire qu'après la Révolution d'Octobre, on avait décidé de mettre la traduction, comme d'autres disciplines, au service des masses populaires. Il s'agissait avant tout de faire connaître les littératures étrangères, qu'elles appartiennent aux Républiques nouvellement créées ou qu'elles soient l'œuvre des pays d'Europe centrale ou occidentale. Ce projet s'était matérialisé en 1919 dans la création par Maxime Gorki de la maison d'édition d'État Littérature universelle (Vsemirnaja Literatura), vaste entreprise de collecte et de diffusion des chefs-d'œuvre étrangers, sous la forme de traductions originales ou révisées. Le comité de rédaction et de lecture était composé des personnalités les plus brillantes de l'époque dans les domaines littéraire et scientifique. On peut citer notamment les poètes Blok et Lozinsky, le critique et historien de la littérature Tchoukovsky, les spécialistes des littératures occidentales Batiouchkov et Smirnov, sans faire l'impasse sur ces remarquables traducteurs qu'étaient Ganzen et Zorgenfrei, aujourd'hui tombés dans l'oubli. C'est ainsi que Littérature universelle édita les œuvres de Voltaire, de Balzac, de Hugo, des frères Goncourt, de Flaubert, de Maupassant et de Daudet. Dans le domaine allemand, Heine, Schiller et Kleist furent à l'honneur. La littérature anglo-saxonne fut représentée par Byron, Coleridge ${ }^{7}$, Dickens, Walter Scott, Mark Twain, George Bernard Shaw, Henry George Wells ou Jack London.

Voici ce qu'écrivait en 1919 Gorki à propos de la maison d'édition Littérature universelle:

Cet ensemble de livres constituera un vaste thésaurus historique et littéraire qui permettra au lecteur de connaître dans le détail la naissance, l'œuvre et la mort des écoles littéraires, le développement de la versification et de la prose, l'interaction des littératures de diverses nations ${ }^{8}$ (traduction personnelle).

Cette merveilleuse entreprise culturelle ne survécut que jusqu'en 1927 et le programme ambitieux se résuma à l'édition de 127 ouvrages sur les 1500 initialement prévus. À l'instar de la NEP dans le domaine économique, on peut véritablement parler de "planification» des œuvres à traduire".

La maison d'édition Littérature universelle s'attela également à former de jeunes talents. Des cours de traduction littéraire y étaient dispensés à d'apprentis traducteurs, sous la supervision de plusieurs maîtres comme Tchoukovsky. Celui-ci publia 
d'ailleurs en 1919 les Principes de traduction littéraire avec la participation de Goumiliov. L’ouvrage comprenait deux parties, la première rédigée par Tchoukovsky et consacrée à la traduction en prose, la seconde écrite par Goumiliov sur la traduction en vers. Il s'agit là sans doute du premier texte traductologique russe - et du premier cours dans ce domaine - après la Révolution d'Octobre, dont l'histoire n'a retenu que le nom de Tchoukovsky. En effet, Goumiliov, le traducteur de Baudelaire et de Rimbaud, fut fusillé en 1921 et toute référence, même livresque ${ }^{10}$, à son nom, désormais interdite. L'œuvre poétique de Goumiliov ne fut d'ailleurs publiée qu'en 1988.

Pour illustrer ce point, il convient de rappeler qu'en 1931, M. P. Alexeev écrivit que la traduction - malgré ses difficultés intrinsèques - est une activité indispensable à la culture du peuple ${ }^{11}$; il traduisit notamment la poésie orientale classique ainsi que les Aphorismes de Confucius. Cette prise de position est fondamentale car, dans la foulée d'Alexeev, nombre de chercheurs focaliseront leur attention sur l'activité traduisante. La traduction est alors véritablement une activité engagée, politique. Bien entendu, celle-ci a toujours été engagée au cours de l'histoire, mais ce qui est inédit, c'est que cet engagement se retrouve de manière explicite dans des manifestes au plus haut niveau de l'État.

Cet engagement se manifestera dans la reconnaissance et la traduction des œuvres nationales des peuples frères, qu'ils appartiennent à l'Union soviétique ou aux pays satellites. Le premier Congrès des écrivains de l'U.R.S.S., tenu en 1934, joua un rôle important sur ce plan. Gorki devait déclarer dans son discours de clôture:

Une connaissance étendue et réciproque des cultures des Républiques sœurs est devenue indispensable [...]. Il faut continuer d'éditer en langue russe, sous forme de recueils, de bonnes traductions des prose et poésie contemporaines des Républiques et des régions nationales ${ }^{12}$ (traduction personnelle).

Dans un courrier adressé au rédacteur d'une revue azerbaïdjanaise des kolkhozes, Gorki devait préciser ses intentions:

[...] il va de soi que [cela] accélérerait de manière importante la création d'une culture socialiste unique, grandiose, redoutable, à même de réformer le monde entier ${ }^{13}$ (traduction personnelle).

C'est ainsi qu'entre 1934 et 1941, on publia en russe deux nouvelles traductions du merveilleux poème de Schota Russthaveli L’homme à la peau de léopard, une traduction de l'épopée arménienne David Sassounk $i^{14}$ et des cycles de la lyrique géorgienne moderne dans les versions de Tikhonov et de Pasternak. Avant d'écrire le Docteur Jivago (1957), Pasternak s'appliqua à traduire Shakespeare: Hamlet (1940), Roméo et Juliette (1942), Antoine et Cléopâtre (1943), Othello (1944), Henri IV (1945), Le Roi Lear (1947) et Macbeth (1950). Il s'attaqua ensuite à Goethe (Faust) et à Schiller (Marie Stuart).

C'est alors qu'apparaît sur le devant de la scène un des plus grands auteurs de l'époque: A. A. Smirnov. Il est le premier théoricien à appliquer à la traduction une méthodologie marxiste et léniniste. Dans un article resté célèbre, Smirnov introduit

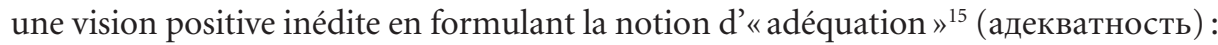
selon lui, les correspondances directes peuvent s'accompagner de substitutions, c'està-dire de compensations (субституты). Dans le domaine littéraire, l'adéquation ne consiste pas à reproduire simplement l'original, mais plutôt à établir une correspon- 
dance esthétique. Lozinsky ne dit pas autre chose en matière de traduction poétique: il faut «créer la même impression que l'original ${ }^{16}{ }$. Voilà donc une véritable équivalence dynamique une trentaine d'années avant les travaux de Nida!

Lozinsky, qui compte parmi les chefs de file de l'acméisme, est l'un des traducteurs les plus doués et les plus éclectiques de l'entre-deux-guerres. On lui doit des traductions aussi réussies et variées que Hamlet et La Nuit des Rois de Shakespeare, le Shah Namè de Firdousi, le Cid de Corneille, l'École de la médisance de Sheridan ou encore des comédies de Lope de Vega comme Le chien du jardinier. Mais surtout, il reçut en 1946 le Prix Staline de littérature pour sa traduction de La Divine Comédie.

L'acméisme est une distanciation par rapport à un symbolisme à bout de souffle et à ses représentants les plus prestigieux, dont Brioussov et Viatcheslav Ivanov ${ }^{17}$. Fondée officiellement en 1912, cette école poétique proclame son programme littéraire dans la revue Apollon. Le premier numéro de 1913 contient notamment le texte fondateur de Goumiliov, "L'héritage du symbolisme et l'acméisme». Il y prône les «principes esthétiques» suivants:

la conjonction de l'univers intérieur de l'homme et d'un sage physiologisme;

l'adhésion à la vie et le perfectionnement des formes artistiques.

Sans le vouloir, Lozinsky fit ainsi naître une opposition fondamentale chez les traductologues de l'époque, entre les partisans d'une approche littéraire et les tenants d'une analyse strictement linguistique. Quoi qu'il en soit, le problème essentiel demeure la notion de traduisibilité, à laquelle s'intéressera notamment le théoricien Finkel ${ }^{18}$.

En Union soviétique donc, la tendance littéraire sera très marquée dans la traductologie de l'époque, avec des articles de Rossel's ${ }^{19}$, Leites et Sobolev entre autres. Ces études minimisent, voire nient l'importance des critères linguistiques en matière de traduction littéraire. Pour ces auteurs, traduire une œuvre littéraire consiste à créer dans une autre langue une nouvelle réalité littéraire, qui reflète la réalité esthétique de l'original. Il s'agit en fait d'une résurgence de la fameuse théorie léninienne du reflet, où la stylistique contrastive est prépondérante par rapport à la linguistique comparée. Et pourtant, les années 1940-1950 ne refléteront que partiellement cet état d'esprit. D'abord, et peut-être en raison de la Seconde Guerre mondiale, on ne traduit que peu les auteurs modernes, a fortiori lorsqu'ils ressortent de l'univers anglo-saxon: aucune traduction de Faulkner, d'Hemingway ou de Maugham. Par ailleurs, l'option résolument littéraire de la traductologie soviétique souffrait d'un défaut chronique: la recherche éperdue d'une langue châtiée, bannissant le langage populaire, l'argot et les vulgarismes, comme pour se démarquer de la littérature occidentale.

Il faudra attendre les années 1950 pour que le rapprochement entre l'Union soviétique et les pays étrangers, notamment en matière de culture, se matérialise dans la traduction. La revue Littérature étrangère (Inostrannaja Literatura), qui voit le jour en 1955, comblera cette lacune. Le nombre de traductions va augmenter de manière considérable et le préjugé relatif au langage populaire sera vaincu. Mais surtout, on abandonnera le formalisme des années 1920-1930. Il faut imaginer combien la censure du langage populaire avait entravé jusqu'aux efforts de théorisation; comment parler d'équivalence, comment rechercher une fidélité sémantique et stylistique avec de tels préjugés? 
La revue Littérature étrangère permit une homogénéisation, une unification du travail, et, par conséquent, une amélioration importante de la qualité des traductions. Jusque-là, il y avait nombre de traducteurs improvisés, méconnaissant la langue - et la culture - de départ et s'appuyant sur des procédés juxtalinéaires qui colorent la traduction d'une teinte originelle. Le procédé juxtalinéaire, qui fait la part belle aux correspondances linguistiques, doit laisser transpirer en permanence l'original sur la langue de la traduction. La traduction ne remplace plus l'original, elle le révèle, au sens photographique du terme. Il s'agit, souvent à l'insu des traducteurs eux-mêmes, d'une traduction pédagogique, utilisable dans les lycées, mais aux dépens de la dimension esthétique. L'Union des écrivains de l'U.R.S.S. organisa dans les années 1950-1960 plusieurs conférences sur la traduction pour condamner - timidement dans un premier temps - le procédé juxtalinéaire.

Mais on aurait tort de croire que le procédé juxtalinéaire se limita en Union soviétique à des traductions littéralistes, comme on le pense généralement en Occident. En réalité, et La poésie de l'Arménie publiée sous la direction de Brioussov en est un exemple, la juxtalinéaire servait avant tout de version intermédiaire visant à rendre le dire de l'auteur original. La juxtalinéaire était suivie de la traduction finale, chargée quant à elle de rendre le vouloir-dire de l'auteur. Elle servait essentiellement aux traducteurs qui ne possédaient qu'une connaissance «linguistique» de la langue de départ, sans en maîtriser les nuances ni les ressources stylistiques cachées; elle avait dès lors une utilité non négligeable, dans la mesure où elle permettait de concilier langue et écriture.

À partir de 1950, l'activité traduisante va connaître un nouvel essor qui se matérialisera dans des dizaines de livres, de recueils et de manuels consacrés à la théorie et à la pratique de la traduction. Cela n'a rien d'étonnant lorsque l'on sait que le même phénomène touche l'Occident. C'est en effet pendant cette décennie que la profession s'organise en Europe occidentale, par la création de la SFT en 1947 et de la FIT en 1953, sous l'impulsion magistrale de Pierre-François Caillé, le traducteur nantais de Autant en emporte le vent, et d'Edmond Cary, l'auteur du remarquable Les grands traducteurs français. L'interprétation ne fut pas en reste, puisque l'AIIC fut créée en 1953 également.

La dimension théorique ne fut pas laissée pour compte; la FIT édita en 1955 sa revue Babel et la SFT publia sans tarder Traduire, lesquelles sont aujourd'hui encore des revues de référence. Dès 1955, le Journal des traducteurs, qui deviendra Meta en 1966, voit aussi le jour.

Enfin, cette théorisation, qui revendiquait pour la traduction une formation sui generis, devait engendrer dans les années 1950 la création de nombre d'écoles de traduction dans le monde: l'ESIT, l'ISIT, Montréal, pour n'en citer que quelquesunes $^{20}$. Pour reprendre l'expression de George Steiner, nous entrons alors dans le «courant moderne $»^{21}$.

On peut dire de manière générale que les années 1950-1960 voient l'éclosion de la traductologie contemporaine, c'est-à-dire libérée de la gangue strictement linguistique qui l'étouffait jusqu'alors. L'Union soviétique connut une évolution tout à fait semblable lorsque le grand théoricien Kachkin apparut sur le devant de la scène. Kachkin laissera son empreinte sur la traductologie russe jusqu'à notre époque. Comme le déclarait Marina Litvinova lors du colloque international de traduction 
organisé par l'Université linguistique d'État de Moscou (MGLU) en avril 2002: «Nous sommes tous des Kachkiniens.»

Dans son ouvrage consacré à la traduction littéraire ${ }^{22}$, Kachkin réussit à concilier les tendances linguistique et littéraire, pour faire entrer la traductologie dans une philologie lato sensu. Il plaidait pour une "traduction réaliste», capable d'intégrer dans sa réflexion les disciplines annexes. Kachkin ouvrit un large chantier sur les principes à appliquer dans l'édition des œuvres traduites. On peut les résumer comme suit:

1. la planification dans les choix des œuvres à traduire;

2. l'ampleur et la diversité du matériau traductif;

3. une grande maîtrise de la traduction, au service de la fidélité notionnelle et de l'originalité artistique de l'œuvre. C'est en cela que réside la justesse de la traduction, laquelle requiert les conditions suivantes:

a) une langue maternelle irréprochable, qui permette de lutter contre les traductions littérales, contre toute contrainte qui puisse peser sur elle;

b) un éventail de procédés à appliquer dans chaque cas concret;

4. une grande créativité dans les traductions et une absence de dogmatisme dans les principes à respecter;

5. la fondation d'un organisme scientifique chargé de coordonner la rédaction et la publication des traductions.

Ce dernier élément est sans doute parmi les plus intéressants. La création des maisons d'édition soviétiques, à commencer par la Littérature universelle, répondit au besoin de publier les traductions de manière plus scientifique, notamment en choisissant le texte original le plus fiable.

Comme on l'aura remarqué, on assiste dans l'ancienne U.R.S.S. à un essor considérable de la traductographie pendant la quarantaine d'années qui ont suivi la Révolution d'Octobre. Et c'est précisément cette explosion de la traductographie qui va fournir un matériau, de moins en moins brut, susceptible de favoriser l'éclosion d'une véritable traductologie soviétique.

Dans les pays d'Europe occidentale ou en Amérique du Nord, on croit généralement que la traductologie est née dans les années 1950, avec les premiers travaux de Nida, de Savory, de Vinay et Darbelnet, d'Edmond Cary ou de Georges Mounin. Ce serait oublier deux éléments essentiels:

- tout d'abord, une traductologie digne de ce nom existe déjà depuis la Renaissance au moins et même avant, selon le critère définitoire adopté;

- parallèlement aux travaux occidentaux qui suivirent la Seconde Guerre mondiale se développèrent à l'Est plusieurs études traductologiques de premier plan, restées malheureusement souvent inconnues. Comme je vais essayer de le montrer plus avant, certaines de ces recherches, et non des moindres, sont antérieures aux publications occidentales.

Ces publications qui apparaissent en Union soviétique à l'aube des années 1920 sont essentiellement des recherches théoriques, historiques et littéraires sur la traduction, englobant de la sorte de nombreuses facettes de l'activité traduisante sans les épuiser toutes. La traduction technique - au sens de «spécialisée» - n'apparaîtra que plus tard dans les préoccupations des théoriciens, mais l'Occident n'échappe pas non plus à cette règle: il faudra attendre 1970 et la publication de La traduction scientifique et technique par Jean Maillot pour que les domaines de spécialité retiennent quelque 
peu l'attention des chercheurs. Cet élément est à mettre en parallèle avec le développement inédit des sciences et des techniques dans la seconde moitié du $\mathrm{xx}^{\mathrm{e}}$ siècle, lequel a eu au moins une vertu: sortir la traductologie de l'emprise des seuls littéraires, comme si seuls les textes des œuvres étaient justiciables d'une approche théorique.

Entre 1920 et 1960, on peut dire que les recherches faites sur la traduction en Union soviétique réunissent les caractéristiques suivantes:

- le lien entre la théorie et la pratique, à savoir entre la philologie et la traduction littéraire, a toujours joué un rôle de premier plan. Les travaux et traductions de Tchoukovsky ${ }^{23}$, de Lozinsky ${ }^{24}$ ou de Marchak ${ }^{25}$ en témoignent;

- la majorité des réflexions théoriques se fondent sur des traductions nouvelles, en font la critique et analysent la méthode de travail appliquée dans chaque cas particulier. Il peut s'agir d'une analyse critique d'une traduction donnée ${ }^{26}$, d'un commentaire ponctuel du traducteur sur son propre travail ${ }^{27}$, d'une postface d'un critique littéraire aux traductions d'un poète ${ }^{28}$ ou encore d'une extrapolation de ses propres principes de traduction ${ }^{29}$. On y retrouve au fil de la lecture des problématiques que l'on pourrait croire ingénument occidentales, comme cette référence implicite à l'objection préjudicielle faite par Batiouchkov en 1920:

[...] aucune traduction, même la plus parfaite, ne peut entièrement remplacer la lecture de l'œuvre littéraire originale $[\ldots]^{30}$ (traduction personnelle).

Ce texte, vieilli aujourd'hui, allait néanmoins influencer les développements ultérieurs de la recherche en traduction, de sorte que la plupart des travaux des années 1920 devaient considérer la fidélité absolue ou «idéale» comme une tâche irréalisable. Par fidélité «idéale», on entendait à l'époque la reproduction de tous les éléments formels de l'original dans leurs mêmes fonctions sémantiques et stylistiques ${ }^{31}$. Pour ce faire, il fallait une conception de la traduction qui aille au-delà de la structure textuelle proprement dite pour considérer l'œuvre à traduire comme une partie d'un ensemble beaucoup plus vaste, incluant le lien avec l'œuvre intégrale de l'auteur. Voici ce que consigna Gorki à cet égard :

Il me semble que, dans la majorité des cas, le traducteur commence son travail dès que le livre est en sa possession, sans l'avoir lu au préalable et sans avoir aucune idée de ses caractéristiques. Mais un seul livre, même s'il a été bien lu, ne permet pas de connaître convenablement la complexité des procédés techniques employés par l'auteur, ses fantaisies lexicales, de déceler ses goûts rythmiques ou le style de sa phrase; autrement dit, d'apprendre toutes les ficelles de son art.

[...] il est indispensable de posséder autant que possible une idée exacte de ce que l'auteur aime et de ce qui le passionne, mais aussi de ce qu'il hait, de ce qui lui est étranger, de ce qu'il préfère taire. Il faut lire tout ce que l'auteur en question a écrit ou, du moins, ses livres les plus reconnus... Le traducteur doit connaitre non seulement l'histoire littéraire, mais aussi l'évolution créatrice de l'auteur. Ce n'est que de la sorte qu'il pourra couler plus ou moins fidèlement l'esprit de chaque livre dans le moule du discours russe ${ }^{32}$ (traduction personnelle).

Il est donc logique que, au début des années 1930, les travaux sur la théorie de la traduction se soient attachés à analyser les techniques de traduction dans leur diversité et à en explorer les écarts par rapport à la fidélité absolue. La méthode utilisée est comparative. L'article écrit par Alexeev en 1931 et où il traite de l'évolution de la traduction en Europe occidentale et en Russie contient un examen de la littérature scientifique de base ${ }^{33}$. 
Juste après la guerre, la traductologie entre, pour quelques années à peine il est vrai, dans une certaine léthargie. On ne voit sortir avant 1950 qu'un seul livre sur la traduction, somme toute assez «politique». Il s'agit des Essais sur les problèmes de la traduction $^{34}$ publiés par Serdioutchenko en 1948. Le relent de patriotisme qui fut le lot de la guerre et de l'après-guerre conduit l'auteur à s'inspirer des vues de Marx, de Engels et de Lénine, ainsi que de la critique démocrate-révolutionnaire russe sur la traduction. Il convient de ne pas oublier que dans les années 1930, l'article de Engels «Comment il ne faut pas traduire $\mathrm{Mar}^{35}{ }^{3}$ ) avait été réédité, ce qui n'avait pas manqué de frapper les esprits.

Les années 1950-1960 furent le théâtre d'une effervescence dans le domaine de l'activité traduisante. On édita à l'époque des dizaines de livres, de manuels et d'articles consacrés à la théorie et à la pratique de la traduction littéraire, sociopolitique, parfois scientifique et technique. Les journaux et les revues des universités partagèrent cet élan. On consacra nombre de thèses aux problèmes linguistiques de la $\operatorname{traduction}^{36}$, à sa didactique ${ }^{37}$ et à son histoire ${ }^{38}$.

Le personnage le plus marquant de cette décennie fut sans conteste Andreï Fédorov. C'est à ce traducteur russe de Saint-Pétersbourg que l'on doit d'avoir élaboré en premier une théorie scientifique de la traduction fondée sur la linguistique. Il publia en 1953 une Introduction à la théorie de la traduction ${ }^{39}$, qui connut un tel succès qu'elle fut de nouveau publiée en 1958, dans une édition refondue. Enfin, une troisième édition vit le jour en 1968, mais avec un titre amendé: Fondements d'une théorie générale de la traduction ${ }^{40}$.

Cet ouvrage, s'il ne comprend pas de linguistique comparée, n'en revendique pas moins l'appartenance de la traduction au domaine de la linguistique. Il s'agit d'un livre polymorphe qui traite à la fois de l'histoire de la traduction, de la typologie des textes et de la traduction poétique. Pour illustrer ce que je viens de dire, je mentionnerai que le chapitre 2 s'intitule Histoire de la traduction avant le marxisme et que le chapitre 3 a pour titre Résumé de l'opinion de Marx, Engels et Lénine sur la traduction.

La polarisation traduction/linguistique se fonde sur l'idée selon laquelle la traduction est d'abord et toujours une opération linguistique. Fédorov élude donc l'extralinguistique, non qu'il l'écarte a priori, pour des raisons de méthode, comme nombre de linguistes avant lui. Il insiste sur la nécessité pour le traducteur d'être armé d'un bagage linguistique à toute épreuve (en philologie, en stylistique et en métrique notamment, ce qui s'explique par le fait que Fédorov est surtout un traducteur littéraire). Le livre envisagera fondamentalement la traduction littéraire, au détriment de la traduction scientifique et technique, dépréciée par l'auteur. Paradoxalement, si l'extralinguistique occupe une place centrale en traduction littéraire, il est passé sous silence. C'est peut-être l'une des raisons pour lesquelles les théoriciens russes ont considéré, du moins dans les années 1920, que toute traduction est impossible. La tendance s'inversera et Fédorov va s'en inspirer pour établir les fondements de sa théorie.

Le travail de Fédorov n'est donc pas, à l'inverse de Vinay et Darbelnet, celui d'un novateur. Il va récupérer les options de certains de ses contemporains et en retirer ce qui lui semble essentiel. On comprend mieux dès lors pourquoi son livre accorde une place importante aux considérations historiques.

Y compris dans l'édition de 1968, Fédorov va soutenir la théorie de la traduisibilité. Il y reprend l'idée des substituts, chère à Smirnov, reconnaissant de la sorte que le traducteur se trouve parfois en présence de «limitations » qui rendent, dit-il, le 
message «intraduisible ou difficilement traduisible ${ }^{41}$. Les substitutions de Smirnov interviennent alors. La notion de traduction idéale reste donc insatisfaite. L'autre idée essentielle dont Fédorov est redevable à Smirnov est celle de l'adéquation (адекватность), qu’il appelle aussi équivalence.

L'adéquation signifie, dans l'esprit de Fédorov, la correspondance fonctionnelle à l'original (équivalence de la communication) et la justification du choix des procédés de traduction (art du style et de la langue $)^{42}$. Il ressort de cette définition que Fédorov considère la notion d'adéquation comme applicable à tout énoncé sans distinction. La typologie des textes ne semble revêtir aucune importance dans sa réflexion.

En défendant sans la moindre réserve la théorie de la traduisibilité, Fédorov soutient ipso facto que l'on peut tout traduire. Il s'oppose ainsi à V.N. Komissarov, lequel préfère distinguer plusieurs niveaux d'équivalence. D'après Komissarov, une traduction est équivalente lorsque les deux textes (l'original et la traduction) possèdent des niveaux de contenu analogues, le terme «contenu» signifiant ici tout ce qu'un texte contient: fonctions sémantiques, stylistiques, etc. Si les divers niveaux d'équivalence semblent innombrables, Komissarov en répertorie cinq de première importance, à savoir les niveaux des signes linguistiques, de l'expression, de la communication, de la description d'une situation et du but de la communication ${ }^{43}$. Il convient de voir dans cette théorie la volonté d'unir les différents modèles théoriques (les théories dénotative, transformationnelle et sémantique) en une théorie unique.

En réalité, le principe de l'équivalence proposé par Fédorov n'est pas aussi monolithique qu'une analyse superficielle ne pourrait le laisser supposer. Pour lui, une traduction équivalente n'est possible que si le traducteur ne se fonde pas sur des éléments isolés, souvent intraduisibles s'ils sont pris séparément, mais sur l'ensemble complexe dans lequel ils s'insèrent et où ils acquièrent leur signification concrète. Reconnaître ce principe permet d'obtenir d'importants résultats théoriques:

1) toute langue évoluée est suffisamment riche pour traduire un contenu qu'une autre langue exprime en unité avec la forme. En outre, les moyens stylistiques de la langue d'arrivée ne servent pas à copier les particularités formelles de l'original, mais à traduire les fonctions stylistiques remplies par les éléments de l'original ${ }^{44}$. De la sorte, ce qui semble impossible pour un élément séparé devient possible pour un ensemble complexe, en révélant et en rendant les fonctions sémantiques et stylistiques des diverses unités qui récusent une reproduction formelle stricte.

2) il est vrai que, dans la pratique de la traduction, on rencontre des cas où un élément de l'original n'est pas rendu entièrement ou est remplacé par une autre forme assez éloignée. Cependant, l'impossibilité de rendre un élément, une particularité de l'original, ne heurte pas le principe de la traduisibilité, puisque celui-ci s'applique à l'œuvre dans son ensemble. Évidemment, l'ensemble ne se comprend pas comme une notion abstraite; il se compose d'éléments concrets qui, néanmoins, ne mènent pas une vie indépendante ni ne se meuvent mécaniquement, mais font partie d'un système, formé de leur combinaison et qui les relie au contenu de l'œuvre. D'où il est possible, pour reprendre l'idée de Smirnov, d'opérer des substitutions et des compensations dans le système du tout. Ainsi la perte d'un élément qui ne joue aucun rôle d'organisation peut ne pas être ressentie au niveau de l'ensemble; cet élément paraît s'y dissoudre ou cède la place à d'autres éléments, parfois même inexistants dans l'original. La corrélation indéfectible entre contenu et forme sert de point de départ pour définir le rôle de chaque élément dans l'original, la nécessité de le 
traduire fidèlement, et aussi la possibilité ou le bien-fondé de son omission ou de sa substitution.

3) cette unité est la condition essentielle de la traduisibilité, en ce sens que cette dernière n'est réalisable que lorsqu'on se trouve en présence d'œuvres offrant une telle unité. Cela signifie que:

[...] le principe de la traduisibilité ne peut s'étendre à des expériences formalistes de tout acabit ni à des artifices vains ou qui dissimulent à dessein le sens supposé. De même, ce principe ne peut s'appliquer à des élucubrations transmentales ${ }^{45}$ de tout genre, à des œuvres qui présentent un déclin de la forme, bref, à tout ce qui ne vaut pas la peine d'être traduit ${ }^{46}$ (traduction personnelle).

Quant à la notion d'adéquation ou d'équivalence, Fédorov avance une définition qu'il qualifie de «rationnelle»:

Une traduction est équivalente si elle rend totalement le contenu sémantique de l'original et si, en regard, elle établit une correspondance fonctionnelle et stylistique parfaite ${ }^{47}$ (traduction personnelle).

Obtenir une traduction équivalente consiste donc à rendre la relation qui existe dans l'original entre le contenu et la forme, en reproduisant les particularités de cette dernière ( $s$ l les conditions linguistiques le permettent) ou en créant des correspondances fonctionnelles à ces particularités. Ce qui suppose l'utilisation de procédés linguistiques qui, tout en possédant une forme distincte des éléments de l'original, rempliraient une fonction expressive analogue dans le système de l'ensemble.

Une traduction équivalente implique par conséquent un certain équilibre entre l'ensemble et le particulier, c'est-à-dire un équilibre entre la transposition du caractère général de l'œuvre et le degré de fidélité atteint par la transposition d'une de ses parties: en effet, des changements apportés à l'original ne pèsent pas de la même façon dans l'ensemble ${ }^{48}$. Autrement dit, certains sont plus importants que d'autres, et le degré de fidélité ou d'écart par rapport à l'original à certains endroits est inévitablement lié à leur importance, peut-être même à la valeur relative d'un mot. Cela signifie que, tout au long de la traduction, l'équivalence peut ne pas exiger un degré unique de fidélité lexicale à l'original. Il ne s'agit donc en aucun cas d'une addition de pertes et de compensations; toute notion quantitative se voit ainsi écartée d'entrée. En d'autres termes, une reproduction minutieuse, rigoureusement exacte, des éléments pris séparément n'implique pas encore une traduction équivalente de l'ensemble, puisque ce dernier n'est pas la simple somme de ces éléments, mais constitue un système homogène.

La conception de l'équivalence, telle que proposée par Fédorov, ne prétend pas à une signification universelle, applicable à toutes les circonstances historiques. Avant tout, elle n'est pas normative: elle n'indique pas comment, à n'importe quelle époque et dans n'importe quel pays, doit être la traduction de n'importe quelle œuvre, quels que soient le pays et l'époque auxquels cette œuvre se rattache. Il s'ensuit que les principes de traduisibilité et d'équivalence ne peuvent être étudiés sans se référer à des situations historiques concrètes. Ainsi par exemple, si l'on traduit au départ d'une langue qui possède une grande richesse lexicale dans une langue dont le lexique est insuffisant pour exprimer certains concepts, certaines nuances et certains sens figurés, il ne faudra pas espérer reproduire de manière équivalente n’importe quel original. L'histoire de la culture connaît de nombreux cas où des obstacles insurmontables se 
sont dressés devant le traducteur; cette situation se produit lorsque la langue de départ est celle d'un peuple plus développé économiquement, politiquement et culturellement, et la langue d'arrivée celle d'un peuple qui n'a pas atteint un tel niveau de développement. Au cours de l'histoire, ces obstacles ont été peu à peu surmontés selon Fédorov, et il érige en norme historique le postulat suivant:

Parallèlement à l'évolution économique, politique et culturelle d'un peuple, à l'enrichissement de sa langue et à l'apparition et au développement de l'activité traduisante, on voit se créer des conditions toujours plus favorables à une traduction équivalente dans une langue donnée et à la réalisation du principe de la traduisibilité ${ }^{49}$ (traduction personnelle).

Fédorov donne ainsi raison à Mounin lorsqu'il formule le concept dynamique (ou dialectique) de la traduisibilité:

L'examen de la traductibilité du russe en français, par exemple, doit ou devra tenir compte de la typologie comparée des deux langues (analyse conduite sur le plan de la pure linguistique descriptive); mais il doit considérer aussi toute l'histoire de tous les contacts entre ces deux langues: traduire du russe en français, en 1960, ne signifie pas la même chose que traduire du russe en français en 1760 (ou même en 1860) quand le premier dictionnaire français-russe (1786) n'existait pas, quand les contacts étaient rares. À partir du XVIII ${ }^{\text {e }}$ siècle chaque traduction du russe, chaque voyage, chaque récit de voyage ajoute une situation commune entre le russe et le français, chaque contact éclairant les suivants, jusqu'à la vogue de Tourguenev, de Tolstoï et de Dostoïevski, laquelle étend ces contacts à des millions de lecteurs français, diminuant chaque fois l'écart entre les situations (non linguistiques et linguistiques) non communes ${ }^{50}$.

Ce concept dialectique revêt à mon sens une importance capitale, car il suit l'évolution de la réalité non linguistique des langues elles-mêmes et des contacts entre elles. Fédorov, s'il privilégie l'approche linguistique, ne le fait pas stricto sensu. Il rejoint sur ce point Georges Mounin. Par ailleurs, de par sa volonté de promouvoir une science de la traduction (perevodovedenie) et d'incorporer son étude scientifique à l'ensemble des disciplines linguistiques, il se situe dans le même courant de pensée que Vinay et Darbelnet, ce qui lui a valu de nombreuses accusations au sein de l'ex-U.R.S.S.

Il est utile de faire remarquer à ce propos que, dans la deuxième édition de son ouvrage (1958), assez remaniée, Fédorov fait des concessions sur ce point. Il y écrit que la traduction peut être étudiée de nombreux points de vue et qu'elle est "polymorphe ${ }^{51}$; que son ouvrage s'attache au côté linguistique de la traduction, ce qui laisse sous-entendre qu'elle recouvre aussi d'autres aspects; que la linguistique ne saurait tout résoudre de la traduction, surtout les problèmes historiques. Enfin, il écrit :

Dans le système des sciences linguistiques, la théorie de la traduction est liée d'une part à la linguistique générale, sur les thèses de laquelle, comme discipline spécialisée, elle doit nécessairement s'appuyer; et d'autre part, elle est liée à la lexicologie, à la grammaire, à la stylistique et à l'histoire des langues en présence, sous tous leurs aspects, dont la phonétique ${ }^{52}$ (traduction personnelle).

On peut se poser la question de savoir si ce recul, assez léger somme toute, est dû à une remise en cause volontaire de ses positions antérieures ou s'il a été dicté par des pressions issues de certaines sphères d'influence. Henri Van Hoof ${ }^{53}$ rappelle avec pertinence qu'avant 1950, la langue faisait partie d'une idéologie en U.R.S.S. Le théoricien Marr 
avait, dans la perspective marxiste, lié l'évolution des langues aux formes de production de la société: dans son esprit, la langue était assujettie à la structure sociale. Quoi d'étonnant à ce que la traduction suive la même voie, celle de la mise en forme idéologique: le traducteur se devait de rechercher non pas l'exactitude linguistique, philologique, mais la conformité aux réalités sociales. Il faut sans doute voir dans la position de Fédorov, partagée d'ailleurs par Staline, la volonté de se libérer de ce carcan antiscientifique.

Au-delà des confrontations entre tenants de l'approche linguistique (Fédorov, Reformatski, Retsker, Barkhoudarov et d'autres) et tenants de l'approche littéraire (Antokolski, Gatchetchiladze...), l'action de Fédorov aura permis la création en U.R.S.S., dès 1956 et dans le cadre de l'Institut Gorki de Moscou, de la première section de traduction dotée de programmes adossés à des critères scientifiques.

Il est nécessaire d'ajouter qu'en matière de traduction automatique, l'Union soviétique participa aux recherches lancées dans les années 1950. L'approche linguistique des problèmes de traduction qui avait prévalu dans le pays jusque-là n'est pas étrangère au phénomène. Il faut citer ici les travaux de Revzin et Rozenzweig, qui tentèrent de concilier les notions de théorie générale de la traduction et les catégories de la linguistique structurale. Leur ouvrage consacré à la traduction automatique ${ }^{54}$ n'a pas encore été, du moins à ma connaissance, traduit en français; il s'agit cependant d'un témoignage intéressant sur l'état de la question à un moment où l'on ne fournissait pas encore de scénarios à la machine. Il est amusant de constater que, dans les années 1960, les recherches sur la traduction automatique voisinent avec des textes très intéressants sur la traduction poétique, sans que le préjugé linguistique ne soit abandonné. Etkind écrira en 1963 son remarquable Poésie et traduction, où l'on peut lire:

La traduction est à la fois un problème littéraire et linguistique. Une théorie qui ignorerait la forme linguistique de l'œuvre désarmerait littéralement le traducteur ${ }^{55}$ (traduction personnelle).

L'empreinte de Fédorov aura marqué l'ensemble de la traductologie soviétique de l'après-guerre. Les deux dernières décennies de l'ère soviétique verront l'apparition de plusieurs travaux dans la droite ligne de leurs prédécesseurs. Il me semble utile de mentionner que la didactique de la traduction rejoint alors les préoccupations des chercheurs. Un juste retour des choses en quelque sorte, dans la mesure où la théorisation, l'objectivation des pratiques, a permis de formaliser un enseignement renonçant désormais à l'artisanat. Les études de Gatchetchiladze ${ }^{56}$, la tentative de classification de Bibikhin $^{57}$ ou la traduisibilité revisitée de Schweitzer ${ }^{58}$ me paraissent s'inscrire dans ce mouvement.

Il y a véritablement une traductologie soviétique, en ce sens qu'un courant de pensée dominant a traversé les écrits du siècle, imposé au départ depuis les plus hautes sphères de l'État, avant que le flambeau ne soit repris par les professeurs d'universités. Il est indéniable que, plus qu'en Occident, la réflexion traduisante s'est développée dans le giron universitaire, certainement après la fin de la Seconde Guerre mondiale. L'hégémonie de la Russie sur les autres Républiques avait imposé la langue russe à l'ensemble du pays et diminué l'impact de la profession sur la théorisation. Il y a donc eu une "académisation" de la traductologie en U.R.S.S., responsable de l'emprise de la linguistique sur la discipline et d'un rejet consécutif de la téléologie. L'enseignement universitaire de la traduction faisait la part belle à la linguistique 
contrastive et était surtout normative; la quête de règles régissant le transfert interlinguistique était omniprésente, ce qui alimentait la recherche.

La traduction, on le sait, ne se limite pas au domaine du linguistique. L'équivalence dynamique, beaucoup plus subtile, se doit d'être à l'œuvre sous la surface textuelle. Pour illustrer cette différence d'approche, je prendrai, en guise de conclusion, deux traductions françaises d'Anna Karénine, qui montrent combien la version peut varier selon l'éclairage adopté. Il s'agit de la mort d'Anna, à la fin du septième chapitre:

«Que fais-je? Où suis-je? Pourquoi?» Anna voulut se redresser, reculer, mais une masse énorme, impitoyable frappa sa tête et l'entraîna sur le dos.

«Seigneur, pardonne-moi!» murmura-t-elle, sentant l'impossibilité de la lutte. Un petit moujik, en marmottant quelques paroles, se pencha sur le fer du marchepied. Et la lumière qui avait éclairé Anna, le livre de sa vie avec ses tourments, ses mensonges, ses douleurs, brilla d'un éclat plus vif, illuminant ce qui, pour la malheureuse, demeurait encore dans les ténèbres - cette lumière trembla, pâlit et s'éteignit à jamais ${ }^{59}$.

(traduction de Marc Semenoff)

«Où suis-je? Que fais-je? Pourquoi?" pensa-t-elle, faisant effort pour se rejeter en arrière. Mais une masse énorme, inflexible, la frappa à la tête et l'entraîna par le dos. "Seigneur, pardonnez-moi!» murmura-t-elle, sentant l'inutilité de la lutte. Un petit homme, marmottant dans sa barbe, tapotait le fer au-dessus d'elle. Et la lumière qui pour l'infortunée avait éclairé le livre de la vie, avec ses tourments, ses trahisons et ses douleurs, brilla soudain d'un plus vif éclat, illumina les pages demeurées jusqu'alors dans l'ombre, puis crépita, vacilla et s'éteignit pour toujours ${ }^{60}$.

(traduction d'Henri Mongault)

Le souffle romanesque de la seconde version est très perceptible dans la dernière phrase, notamment dans le rythme de trois qui rend bien la flamme de la vie qui s'éteint. La première version est plus hésitante, voire chaotique, avec la relative «ce qui », la répétition du mot «lumière » et le mauvais placement syntaxique de l'incise «pour la malheureuse».

La version de Semenoff est corsetée dans l'habit linguistique de l'original, alors que la version de Mongault libère l'émotion contenue dans le texte. Le premier phénomène est assez illustratif de l'époque soviétique; la prise en compte du lecteur dans toute sa personnalité était une tâche particulièrement ardue, dans la mesure où les traductions répondaient à un canon imposé d'en haut et passé par le crible universitaire. C'est ce cachet universitaire, conjugué à un marché de la traduction centripète par la force des choses, qui devait imprimer sa marque à la traductologie soviétique qu'il s'agisse de la manière de traduire ou, a fortiori, du choix des originaux.

La centralisation du pouvoir après 1917 et la production d'une littérature de masse, y compris en matière de traduction, devaient obligatoirement conduire à une imposition de règles émanant du sommet de l'État, c'est-à-dire à une traductologie soviétique. La chute du pouvoir soviétique et l'émancipation des anciennes Républiques sœurs vont inévitablement donner lieu à l'avenir à de nouvelles conceptions traduisantes.

\section{NOTES}

1. «Traductibilité» est le vocable utilisé par Georges Mounin (Les Problèmes théoriques de la traduction, Paris, Gallimard, 1963, p. 277). D’autres auteurs préfèrent le terme «traduisibilité ». À la traduisibilité s'oppose l'intraduisibilité, ce que Mounin et Ladmiral appellent «objection préjudicielle». 
2. Voir Chr. Balliu: «Tendances actuelles de la traduction en Union soviétique », Équivalences, revue de l'Institut supérieur de Traducteurs et Interprètes (ISTI), Bruxelles, 1979, vol. 10/1-2, p. 45-49.

3. La poésie de l'Arménie, éditée en 1916 sous la direction de Brioussov, est faite de transcriptions et de juxtalinéaires, dues notamment au fait que Brioussov ne dominait pas la langue arménienne. On sait que les carences linguistiques sont souvent le prélude à la régression littéraliste.

4. On peut mentionner Balmont, le traducteur de Schelling et de Calderón, ainsi que Viatcheslav Ivanov, le traducteur d'Eschyle et de Pétrarque.

5. Le syntagme « réalisme socialiste» fut créé en 1932.

6. Il suffit de rappeler les efforts déployés par Edmond Cary entre autres dans le domaine de la formation et de la professionnalisation.

7. Cristabel de Coleridge fut traduit par Guéorgui Ivanov (à ne pas confondre avec Viatcheslav Ivanov) pour les éditions Littérature universelle en 1922.

8. M. Gorki, Articles épars de critique littéraire, Moscou, Goslitizdat, 1941, p. 279.

9. Ce phénomène n'est pas nouveau dans l'histoire. Le choix des originaux, dicté par les dirigeants, se retrouve déjà, et c'est loin d'être le seul cas, au XIV ${ }^{\mathrm{e}}$ siècle français sous Charles V le Sage.

10. Le texte de Goumiliov sur la traduction en vers (1919) a été repris dans le recueil Perevod-sredstvo vaimnogo sblijenija narodov [La traduction, moyen de rapprochement entre les peuples].

11. M. P. Alexeev, «Le problème de la traduction littéraire», Sbornik rabot Irkutskogo Gosudarstvennogo Universiteta, t. VIII, 1, Irkoutsk, 1931, p. 157-173.

12. M. Gorki, Euvres, t. 27. Moscou, Goslitizdat, 1953, p. 342.

13. M. Gorki, Euvres, t. 30, Moscou, Goslitizdat, 1955, p. 365-366.

14. Le travail de Brioussov en matière de poésie arménienne est remarquable. Il s'assura la collaboration de Balmont, de Viatcheslav Ivanov et même de Blok pour éditer en 1916 La poésie de l'Arménie.

15. A. A. Smirnov, «Traduction», Literaturnaja Enciklopedija, t. 8, 1934, p. 527.

16. Družba narodov [Amitié des peuples], 1955, nº 7, p. 166.

17. Les symbolistes russes s'occupaient beaucoup de traduction. Ainsi Brioussov et Viatcheslav Ivanov traduisirent-ils L'Art de Théophile Gautier (1852), le premier en 1897 et le second en 1910. Ce poème était devenu le porte-drapeau du Parnasse russe. Annenski, spécialiste des lettres classiques et de la poésie française, nous a légué une traduction complète d'Euripide. À la différence de tous les autres poètes symbolistes russes, Andreï Biély, l'auteur de l'extraordinaire Pétersbourg (1916), ne traduisit rien.

18. Voir son bel article "Quelques problèmes de la théorie de la traduction", Travaux scientifiques de l'Institut pédagogique d'État des langues étrangères de Kharkov, t. I, 1939, p. 59-82.

19. V. M. Rossel's, "Dans l'atelier du traducteur», Tetradi perevodčika [Cahiers du traducteur], no 3, Moscou, 1966.

20. Il est intéressant de noter à ce propos que l'école de traduction et d'interprétation (ETI) de l'Université de Genève fut fondée en 1941 par le Belge Antoine Velleman.

21. George Steiner, Après Babel, traduction de Lucienne Lotringer, Paris, Albin Michel, 1978, p. 225-226.

22. I. A. Kachkin, La méthode et l'école soviétiques de traduction littéraire, Znamja, 1954, n 10, p. 152.

23. K. I. Tchoukovsky, «Les traductions prosaïques», Principy hudožestvennogo perevoda [Principes de traduction littéraire], 1919.

24. M. L. Lozinsky traduisit, avec des commentaires remarquables, des chansons populaires serbes, rassemblées par Vuk Karadžič. Elles ne furent publiées que très tard.

25. Aujourd'hui encore, ses traductions de Shakespeare, Blake et Burns jouissent des faveurs du public. Voir notamment: S. Marchak, "Sonnets de Shakespeare traduits par S. Marchak», Sovetskij Pisatel" [L'écrivain soviétique], Moscou, 1949.

26. Par exemple, I. A. Kachkin, «Faux principe et résultats inacceptables. Du mot à mot dans les traductions russes de Charles Dickens", Le critique littéraire, 1936, nº 5.

27. Notamment M. P. Stoliarov, «L'art de la traduction de la prose littéraire», Le critique littéraire, 1939, $\mathrm{n}^{\text {os }}$ 5-6.

28. M. M. Morozov, Choix d'articles et de traductions, Moscou, Goslitizdat, 1954.

29. N. M. Lioubimov, «La traduction, un art», Masterstvo Perevoda [L'art de la traduction], 1963.

30. F. D. Batiouchkov, Principes de traduction littéraire, $2^{\mathrm{e}}$ éd., Saint-Pétersbourg, 1920, p. 10.

31. Voir à ce sujet l'avant-propos d'Andreï Biély à la traduction du «Chant de l'amour et de la mort du cornette Christophe Rilke». R. M. Rilke, Almanach «Svitok», 1924, n 3.

32. Cité dans K. I. Tchoukovsky, Vysokoe Iskusstvo [Grand art], Moscou, éd. Iskusstvo, 1964, p. 349-350 (annexes). Dans ce même ouvrage, Tchoukovsky critique abondamment les erreurs de traduction dans le Shelley de Balmont, qu'il appelle pour l'occasion «Shel-mont». 
33. M. P. Alexeev, ibid.

34. G. P. Serdioutchenko, Očerki o problemah perevoda [Essais sur les problèmes de la traduction], Nal'čik, 1948.

35. F. Engels, Euvres complètes, t. XVI, $1^{\mathrm{e}}$ partie, 1937. Cet article date en réalité de 1885.

36. Par exemple: J. I. Retsker, Signification grammaticale et stylistique des constructions absolues en anglais moderne, Moscou, 1953 (le chapitre IV est consacré aux problèmes de la traduction); G. P. Chekurov, Quelques problèmes de la traduction du russe en français (travail fondé sur des traductions du poème de Gogol Les âmes mortes), Léningrad, 1956.

37. Par exemple: S. N. Bondar, Méthode utilisée dans un Institut supérieur qui n'est pas un Institut de langues, pour faire des traductions en langue maternelle, Léningrad, 1951. À ce propos, il n'est pas inutile de relire ce que Ladmiral écrivait de la «traduction pédagogique» dans Traduire: théorèmes pour la traduction, Paris, Payot, 1979.

38. Par exemple: N. E. Rudenko, Gogol dans les traductions françaises, Lvov, 1955. Ce livre étudie l'histoire des liens littéraires entre la Russie et la France de 1840 à 1860.

39. A. V. Fédorov, Vvedenie v teoriju perevoda, Moscou, Institut des littératures en langues étrangères, 1953.

40. Osnovy obščej teorii perevoda (lingvističeskij očerk), Moscou, éd. Vysšaja Škola, 1968.

41. $\quad 3^{e}$ éd., 1968, p. 146.

42. Ibid., p. 149.

43. V. N. Komissarov, Slovo o perevode [De la traduction], Moscou, 1973, p.62.

44. A. V. Fédorov, ibid., p.144.

45. On ressent nettement la critique implicite à l'encontre des formalistes, notamment Chklovski et Jakobson. Pour ceux-ci, aucune différence ne démarque la prose de la poésie: les deux sont issues de la nature même des signifiants. La zaumnyj jazyk (langue transmentale) compose une esthétique textuelle de pure sonorité, déplaçant le sens (smyslovoj sdvig) pour créer des mots arbitraires, en fonction de parangons phoniques. Les futuristes avaient déjà accordé une importance toute particulière au processus métonymique, si fréquent en traduction; ils aimaient à remplacer la signification référentielle par la signification réelle. Enfin, Gogol, Leskov et d'autres utilisent dans certains récits des clefs de production textuelle que l'on retrouvera dans la prose transmentale. Il est utile de lire le texte théorique de Chklovski: «Iskusstvo kak priem» ["L'Art comme objet»], O teorii prozy [ La théorie de la prose], Federacija, Moscou, 1929.

46. A. V. Fédorov, ibid., p.147.

47. Ibid., p. 151.

48. On retrouve la conception de la traduction exprimée dans le De optimo genere oratorum (46 avant J.-C.) où Cicéron préconise de «peser la somme en bloc».

49. A.V. Fédorov, ibid., p.153.

50. G. Mounin, Les problèmes théoriques de la traduction, Paris, Gallimard, 1963, p. 277.

51. A. V. Fédorov, $2^{\text {e }}$ éd., p. 15.

52. Ibid., p. 21.

53. H. Van Hoof, Histoire de la traduction en Occident, Louvain-la-Neuve, Duculot, 1991, p. 363.

54. I. I. Revzin et V. J. Rozenzweig, Osnovy obščego i mašinnogo perevoda [Les fondements de la traduction générale et automatique], Moscou, 1964. Pendant la guerre froide, des recherches de ce type furent également menées aux Etats-Unis, essentiellement à des fins d'espionnage militaire ou industriel.

55. E. G. Etkind, Poezija i perevod, Moscou-Léningrad, Soveckij Pisatel', 1963, p. 135.

56. G. R. Gatchetchiladze, "Théorie de la traduction littéraire et préparation des jeunes traducteurs", Traduction littéraire - Interaction et enrichissement mutuel des littératures, Erevan, éd. Université d'Erevan, 1973.

57. V. V. Bibikhin, «Pour une définition de l'essence de la traduction», Tetradi perevodčika [Cahiers du traducteur], $\mathrm{n}^{\circ}$ 10, Moscou, 1973.

58. A. D. Schweitzer, Perevod i lingvistika [Traduction et linguistique], Moscou, 1973.

59. L. N. Tolstoï, Anna Karénine, traduit par Marc Semenoff, Paris, éd. Baudelaire, 1965, p. 541-542.

60. L. N. Tolstoï, Anna Karénine, traduit par Henri Mongault, Paris, Gallimard, Folio, 1952, p. 391-392. 